\title{
Prevalência de Blastocystis spp. provenientes de amostras fecais de moradores de dois biomas mato-grossenses
}

\author{
Wilma Nunes Martins Zorzan ${ }^{1}$ (D), Luciana Melhorança Moreira ${ }^{1}$ (D), Samuel Laudelino da Silva ${ }^{1}$ (D), \\ Solange Kimie Ikeda Castrillon ${ }^{1}$ (D), Marília Ferreira Lima ${ }^{1}$ (D), Maria Eugenia Villaruel da Silva ${ }^{1}$ (D), \\ Stephany Seraglio Santos Cabral ${ }^{1}$ (D), Lucas França de Barros ${ }^{1}$ (D), Jeffrey Jon Shaw ${ }^{2}$ (D), Antonio Francisco \\ Malheiros ${ }^{1 \star(D)}$

\footnotetext{
${ }^{1}$ Faculdade de Ciências Agrárias e Biológicas, Universidade do Estado do Mato Grosso, Campus JANE VANINI, Av. São João, 563, Cavalhada I, 78216-060

${ }^{2}$ Departamento de Parasitologia, Instituto de Ciências Biomédicas, Universidade de São Paulo, Butantã, São Paulo, SP, 05508000
} \\ * Autor para correspondência: malheiros@unemat.br
}

Recebido em 19 de junho de 2019.

Aceito em 23 de setembro de 2020.

Publicado em 30 de setembro de 2020.

Resumo - Este trabalho teve por objetivo avaliar o percentual de positividade de Blastocystis spp. na população de quatro municípios de dois biomas do estado de Mato Grosso. As coletas de fezes foram realizadas nos municípios de Barão de Melgaço, Cáceres, Nova Xavantina e Cuiabá, nos biomas Pantanal e Cerrado. Foram coletadas 708 amostras fecais, o grupo amostral foi dividido em duas categorias sendo 350 provenientes do grupo I, o qual compreendia uma população composta por integrantes com idade entre 5 a 15 anos e 358 provenientes do grupo II, composto por integrantes com idade de 16 anos acima, destas 17,93\% estavam positivas para Blastocystis spp, sendo que 11,15\% eram amostras de pertencentes ao primeiro grupo e 6,77\% referentes ao segundo grupo. Barão de Melgaço foi o município que apresentou a maior positividade (25,5\%), e Cáceres a menor (7\%). O bioma Cerrado apresentou um percentual maior de amostras positivas na população geral $(21,10 \%)$ em relação ao Pantanal (15,5\%). Houve diferença significativa para o percentual de positividade com relação à faixa etária, não havendo diferença entre os indivíduos por sexo ou por biomas. Este trabalho possibilitou um maior conhecimento sobre a realidade mato-grossense quanto à infecção por Blastocystis spp.

Palavras-chave: Blastocystis spp.. Prevalência. Epidemiologia.

\section{Prevalence of Blastocystis spp. from fecal sample of residents in two biomes in Mato Grosso}

\begin{abstract}
This study aimed to evaluate the percentage of positivity of Blastocystis spp. in the population of four municipalities in two biomes in the state of Mato Grosso. Stool collections were performed in the municipalities of Barão de Melgaço, Cáceres, Nova Xavantina and Cuiabá, in the Pantanal and Cerrado biomes. 708 fecal samples were collected, the sample group was divided into two categories, 350 of which came from group I, which comprised a population composed of members aged 5 to 15 years and 358 from group II, composed of members aged 16 years above, of these $17.93 \%$ were positive for Blastocystis spp., with $11.15 \%$ being samples belonging to the first group and $6.77 \%$ referring to the second group. Barão de Melgaço was the municipality with the
\end{abstract}


highest positivity (25.5\%), and Cáceres the lowest (7\%). The Cerrado biome had a higher percentage of positive samples in the general population (21.10\%) compared to the Pantanal (15.5\%). There was a significant difference for the percentage of positivity in relation to the age group, with no difference between individuals by sex or biomes. This work enabled greater knowledge about the reality of Mato Grosso regarding infection by Blastocystis spp.

Keywords: Blastocystis spp.. Prevalence. Epidemiology.

\section{La prevalencia de Blastocystis spp. de muestras fecales de residentes de dos biomas de Mato Grosso}

Resumen - Este estudio tuvo como objetivo evaluar el porcentaje de positividad de Blastocystis spp. en la población de cuatro municipios en dos biomas del estado de Mato Grosso. Las recolecciones de heces se realizaron en los municipios de Barão de Melgaço, Cáceres, Nova Xavantina y Cuiabá, en los biomas Pantanal y Cerrado. Se recolectaron 708 muestras fecales, el grupo de muestra se dividió en dos categorías, de las cuales 350 provenían del grupo I, que comprendía una población compuesta por integrantes de 5 a 15 años y 358 del grupo II, compuesto por integrantes con más de 16 años, de estos el 17,93\% fueron positivos para Blastocystis spp., siendo el 11,15\% muestras pertenecientes al primer grupo y el 6,77\% referentes al segundo grupo. Barão de Melgaço fue el municipio con mayor positividad $(25,5 \%)$ y Cáceres la menor $(7 \%)$. El bioma Cerrado tuvo un mayor porcentaje de muestras positivas en la población general $(21,10 \%)$ en comparación con el Pantanal $(15,5 \%)$. Hubo una diferencia significativa para el porcentaje de positividad en relación al grupo de edad, sin diferencia entre los individuos por sexo o biomas. Este trabajo permitió conocer mejor la realidad de Mato Grosso en cuanto a la infección por Blastocystis spp.

Palabras clave: Blastocystis spp.. Prevalencia. Epidemiologia.

\section{Introdução}

Blastocystis spp. é um protozoário intestinal, anaeróbico membro da família Stramenopiles (Silberman et al. 1996; Martins et al. 2007; Eymael et al. 2010), comumente encontrado em amostras de fezes de diversas espécies animais, incluindo humanos. Seu ciclo é compreendido como contaminação fecal-oral (Cimerman et al. 2003), com início do ciclo pela ingestão de alimentos e água contaminados pela forma cística, no intestino grosso ocorre a excistação e o parasita se desenvolve em formas vacuolares, a encistação ocorre durante a passagem ao longo do intestino grosso e é depositada nas fezes (Tan 2008). Este tipo de ciclo contribui para uma maior prevalência em locais onde há precárias condições sanitárias (Nolla e Cantos 2005).

É um protozoário polimórfico, com distribuição mundial (Jelinek et al. 1997; Tan 2008) e frequência elevada, sobretudo em países tropicais e em desenvolvimento (Cheng et al. 2006), como na Venezuela onde a prevalência no estado de Anzoátegui foi de 46,9\% (Velásquez et al. 2005), na Líbia, com prevalência em pacientes menores de 18 anos de 29,4\% (Abdulsalam et al. 2013) e no Senegal, onde a prevalência em crianças chegou a 100\% (El Safadi et al. 2014). Pelos métodos convencionais de exame parasitológico de fezes, é um dos parasitas mais encontrados (Devera 1999) e também o agente mais comum na diarreia do viajante (Silva 2006; Alarcón et al. 2007; Takizawa et al. 2009).

A prevalência pode variar entre 30 e 50\% nos países em desenvolvimento e uma prevalência de 1,5\% a 10\% nos países desenvolvidos (Stenzel E BOREHAM 1996). 
Diversos estudos têm registrado uma elevada frequência de Blastocystis spp. na população, sem conseguir, no entanto, determinar de fato o potencial patogênico desse protozoário (Martins et al. 2007, Abdulsalam et al. 2013, El Safadi et al. 2014, Osman et al. 2015).

A patogenicidade deste parasita em humanos está longe de ser definida, a maioria dos estudos falhou em demonstrar uma correlação significante entre a prevalência do Blastocystis spp. com os sintomas (Tan 2008, Khademvatan 2017), pois é frequentemente encontrado tanto em indivíduos com sintomas entéricos quanto em indivíduos aparentemente saudáveis e assintomáticos (Silberman et al. 1996, Eymael, Schuh e Tavares 2010, Abdulsalam et al. 2013).

Além disso, o próprio ciclo biológico deste parasita também é cercado por controvérsias, já que ainda não foi completamente elucidado, três modos de reprodução foram confirmados: fissão binária, plasmotomia e brotação (Zhang et al. 2012). Fatores de risco reportados para aquisição de Blastocystis spp. incluem viagem a áreas tropicais, consumo de água não tratada e cuidado de animais (Amato Neto et al. 2003). O Blastocystis spp. é frequentemente relatado em turistas que voltam de países em desenvolvimento (Silva 2006), mas também é encontrado em amostras fecais de indivíduos imunocompetentes e imunossuprimidos (Amato Neto et al. 2003, Eymael, Schuh e Tavares 2010, Amâncio et al. 2012).

Alguns fatores podem colaborar para a dificuldade dos estudos em determinar a patogenicidade do micro-organismo, tais como: a falta de critérios padronizados para o diagnóstico, uma limitação natural da infecção, variabilidade genética, tempos de geração de isolados, diferentes antecedentes do hospedeiro como status imunológico, a existência de estados assintomáticos e a possibilidade que pode haver cepas virulentas e não virulentas do organismo (Amato Neto et al. 2003, Audebert et al. 2016).

No Brasil, a presença de Blastocystis spp. já foi relatada em alguns estudos, seja em portadores hígidos ou em imunodeprimidos (Nascimento e Moitinho 2005; Aguiar et al. 2007; Martins et al. 2007, Cabrine-Santos et al. 2016, Zanetti et al. 2020), mas especialmente no território mato-grossense são poucos e incipientes os estudos que revelam a prevalência deste parasita, o que corrobora para o desconhecimento das peculiaridades inerentes ao protozoário. Averigua-se que seu potencial zoonótico, ou seja, sua capacidade de infectar diferentes espécies incluindo a habilidade de infectar humanos.

O Brasil, de acordo com o Ministério do Meio Ambiente, apresenta em sua distribuição territorial seis Biomas de características diferentes, enquanto no Estado de Mato Grosso há a presença de três distintos Biomas no seu território. O presente estudo focou em investigações nos dois biomas mais abrangentes do Estado, considerando-se as diversas características ambientais, sejam aquelas relacionadas à vegetação, clima, presença de animais, entre outras, e também com relação ao desenvolvimento e características culturais.

Segundo Wilkinson et al. (2018), a fragmentação dos habitats pode gerar áreas de maior contato que contribuem para novas conexões entre os habitats naturais e os ambientes antrópicos. Essas interações podem favorecer a aproximação entre os reservatórios, vetores e hospedeiros, aumentando as possibilidades de transmissão. Existem evidências que associam alterações de paisagens com emergência de várias doenças (Patz et al. 2004). Estima-se que mais de 60\% das doenças infecciosas humanas já conhecidas e mais de 75\% de outras emergentes serão derivadas de zoonoses. Quanto maior a proximidade humana com outros animais, maior será a exposição aos agentes infecciosos a diferentes agentes infecciosos (Jones et al., 2008). 
Cada vez mais temos evidências de que a qualidade da saúde humana, dos animais e dos ecossistemas está inexoravelmente interligada (Costanza, 2020). Este estudo teve por objetivo avaliar o percentual de positividade de Blastocystis spp. na população de quatro municípios de dois biomas do Estado de Mato Grosso.

\section{Material e métodos}

Realizou-se um estudo parasitológico descritivo com amostragem aleatória e delineamento transversal ou de corte-transversal, a fim de se obter o percentual de positividade para Blastocystis spp. na população de quatro cidades do Estado de Mato Grosso: Barão de Melgaço e Cáceres, situadas no bioma Pantanal e Nova Xavantina e Cuiabá, situadas no bioma cerrado.

As coletas de amostras de fezes foram realizadas nos meses de fevereiro a novembro de 2014. A população do estudo foi dividida em dois grandes grupos, sendo padronizada a coleta de 100 amostras para o Grupo I e 100 amostras para o Grupo II em cada um dos municípios. Para composição do grupo I foram consideradas as pessoas na faixa etária entre 5 a 15 anos e para o grupo II foram considerados todos os indivíduos com idade maior ou igual a 16 anos, independente do sexo. No município de Cuiabá não foi possível atingir o número planejado de indivíduos frente à dificuldade de acesso as pessoas que se dispuseram a participar da pesquisa, mas optaram por não entregarem as amostras fecais ou não foram encontradas no domicilio nos dias pactuados ou subsequentes para recolhimento das amostras e, portanto não foi possível a análise laboratorial e desse modo a coleta encerrou-se com amostras de 58 crianças e 50 adultos, num total de 108 pessoas. Para obtenção de uma amostragem heterogênea da população foi estabelecido um plano de ação, junto aos gestores e agentes comunitários de saúde, que abrangesse a maior parte da população do município adstrita às unidades de saúde da família. Definiu-se que o total de amostras padronizadas fosse dividido pelo total de unidades de saúde que o município dispunha a única exceção a este princípio foi o município de Cuiabá, que por apresentar um grande número de unidades de saúde da família, foi delimitado pelos gestores uma unidade para cada região da cidade (norte, sul, leste, oeste) e uma unidade na zona rural, totalizando cinco unidades de saúde para este município.

Para levantamento de faixa etária, sexo e características socioambientais dos indivíduos em estudo, foram realizadas entrevistas através de um questionário semiestruturado, contemplando indicadores demográficos e socioeconômicos. As crianças foram entrevistadas acompanhadas por um de seus pais ou responsável. Para a coleta das fezes, cada entrevistado recebeu frascos descartáveis estéreis (coletor universal), devidamente identificados com nome, idade, sexo, unidade de saúde a qual pertence e zona de habitação.

As amostras fecais coletadas foram encaminhadas para análise no Laboratório de Parasitologia da Universidade do Estado de Mato Grosso-UNEMAT.

A análise das amostras foi realizada utilizando a técnica coproparasitológica de sedimentação espontânea - método de Hoffmann, para a identificação dos parasitas - cerca de $5 \mathrm{~g}$ de fezes foram dissolvidas em $50 \mathrm{~mL}$ de Água corrente limpa em frasco pequeno, misturado vigorosamente e posteriormente filtradas com parasitofiltro, utilizando um cálice de sedimentação e deixadas em repouso por cerca de duas horas. Após a sedimentação a amostra foi retirada do fundo do vértice do cálice com uma pipeta e examinada ao microscópio adicionando uma gota da solução de lugol (De Carli, 2007). 
As amostras que não puderam ser analisadas nesse período, passavam por centrifugação e o sobrenadante era desprezado e preenchido com formol a $10 \%$ na proporção de três para um, permanecendo em refrigerador em temperatura de 2 a $8^{\circ} \mathrm{C}$, até a leitura (De Carli, 2007).

Os dados identificados foram analisados por meio do Programa Estatístico R 3.0.1, utilizando o teste Qui-quadrado de independência, com nível significância de 5\% ( $\mathrm{p}<0,05)$, para comparar a presença e ausência de positividade para Blastocystis spp. com as variáveis independentes (sexo, idade, bioma).

Este estudo foi submetido e aprovado pelo comitê de ética em pesquisa do Instituto de Ciências Biomédicas da USP sob o parecer no 310.501, estando em consonância com a Resolução 466/2012 do Ministério da Saúde. Os participantes que aceitaram o convite assinaram o termo de consentimento livre e esclarecido e o termo de assentimento (menores de idade), a eles foi garantido o sigilo das informações e destacado o direito da não participação ou suspensão da mesma em qualquer momento.

\section{Resultados}

Neste estudo 708 amostras foram coletadas e analisadas, sendo 350 amostras provenientes dos adultos e 358 de crianças. Do total dos participantes da pesquisa, 43,23\% correspondiam a indivíduos do sexo masculino, enquanto $56,77 \%$ eram do sexo feminino. $79,8 \%$ residiam na zona urbana dos municípios, enquanto 20,2\% eram moradores da zona rural. Quanto à escolaridade e renda familiar, 70,53\% possuíam ensino fundamental incompleto e 48,30\% viviam com uma renda inferior a um salário mínimo.

Sobre o tipo de moradia e hábitos dos entrevistados, 96,04\% apresentaram casas de alvenaria; $83,62 \%$ com quintal em terra, enquanto $13,13 \%$ cimentaram este ambiente; $68,64 \%$ relataram possuir algum animal doméstico e 43,23\% mantinham algum tipo de contato com animais de vizinhos. A análise de dados demonstrou que 57,07 \% dos entrevistados consumiam água das torneiras provenientes da rede de abastecimento pública, 25,14\% utilizavam a água procedente de poços ou cisternas e 16,10\% utilizavam a água mineral procedente da aquisição particular em distribuidoras ou ainda em minas particulares de sua propriedade.

Com relação ao destino do esgoto doméstico produzido, observou-se que 85,03\% dos entrevistados não tinham acesso a rede de tratamento de esgoto, fazendo uso da fossa séptica. Quanto ao destino do lixo $86,44 \%$ dos entrevistados tinham acesso à coleta pública realizada pelas prefeituras, enquanto $12,29 \%$ queimavam o lixo produzido em suas residências.

Das 708 amostras analisadas, 17,93\% foram positivas para o Blastocystis spp. O município de Barão de Melgaço foi o que apresentou o maior percentual de positividade para Blastocystis spp. em sua população, 25,5\% entre a população geral, sendo $29 \%$ entre integrantes do Grupo I e $22 \%$ entre os do Grupo II. Já Cáceres foi o que apresentou os menores percentuais de positividade, 7\% na população geral, sendo $8 \%$ entre as crianças e 6\% entre os adultos (Tabelas 1).

Quando o percentual de positividade é analisado através da ótica de diferenciação por biomas observa-se que no Cerrado o percentual de positividade das amostras na população geral foi de $21,10 \%$ enquanto no Pantanal foi de 15,5\%. Com relação à positividade, entre os grupos analisados observamos que entre o grupo I cuja população é representada por crianças e adolescentes com idade inferior a 16 anos a positividade foi de $42 \%$ no Cerrado e $37 \%$ no Pantanal, porém na população do Grupo II, pessoas com idade de 16 anos acima, esta diferença não foi observada, sendo 13,33\% no Cerrado e 14\% no Pantanal (Tabelas 1). 
Tabela 1. Percentual de positividade para Blastocystis spp. na população geral, no grupo I (integrantes com idade entre 5 a 15 anos) e no grupo II (integrantes com idade de 16 anos acima) de indivíduos participantes da pesquisa nos municípios de Barão de Melgaço, Cáceres, Nova Xavantina e Cuiabá - MT, 2014.

\begin{tabular}{|c|c|c|c|c|c|c|c|c|c|}
\hline \multirow[b]{2}{*}{ Município } & \multicolumn{3}{|c|}{$\begin{array}{c}\text { Percentual de Positividade } \\
\text { geral }\end{array}$} & \multicolumn{3}{|c|}{$\begin{array}{l}\text { Percentual de Positividade no } \\
\text { Grupo I }\end{array}$} & \multicolumn{3}{|c|}{$\begin{array}{c}\text { Percentual de Positividade no } \\
\text { Grupo II }\end{array}$} \\
\hline & $\begin{array}{l}\text { Amostras } \\
\text { Positivas }\end{array}$ & $\%$ & $\begin{array}{c}\text { Total de } \\
\text { amostras }\end{array}$ & $\begin{array}{l}\text { Amostras } \\
\text { Positivas }\end{array}$ & $\%$ & $\begin{array}{c}\text { Total de } \\
\text { amostras }\end{array}$ & $\begin{array}{l}\text { Amostras } \\
\text { Positivas }\end{array}$ & $\%$ & $\begin{array}{c}\text { Total de } \\
\text { amostras }\end{array}$ \\
\hline $\begin{array}{l}\text { Barão de } \\
\text { Melgaço }\end{array}$ & 51 & 25,5 & 200 & 29 & 29 & 100 & 22 & 22 & 100 \\
\hline Cáceres & 14 & 7 & 200 & 8 & 8 & 100 & 6 & 6 & 100 \\
\hline $\begin{array}{l}\text { Nova } \\
\text { Xavantina }\end{array}$ & 42 & 21 & 200 & 29 & 29 & 100 & 13 & 13 & 100 \\
\hline Cuiabá & 20 & 18,51 & 108 & 22,41 & 13 & 58 & 7 & 14 & 50 \\
\hline Bioma & $\begin{array}{l}\text { Amostras } \\
\text { Positivas }\end{array}$ & $\%$ & $\begin{array}{c}\text { Total de } \\
\text { amostras }\end{array}$ & $\begin{array}{l}\text { Amostras } \\
\text { Positivas }\end{array}$ & $\%$ & $\begin{array}{l}\text { Total de } \\
\text { amostras }\end{array}$ & $\begin{array}{l}\text { Amostras } \\
\text { Positivas }\end{array}$ & $\%$ & $\begin{array}{l}\text { Total de } \\
\text { amostras }\end{array}$ \\
\hline Cerrado & 62 & 21,10 & 308 & 26,58 & 42 & 158 & 20 & 13,33 & 150 \\
\hline Pantanal & 65 & 15,5 & 400 & 18,5 & 37 & 200 & 28 & 14 & 200 \\
\hline
\end{tabular}

Observa-se que há diferença estatística entre a variável dependente positividade de Blastocystis spp. quando analisada com as variáveis independentes bioma, sexo e a idade dos indivíduos, porém individualmente não houve significância estatística em relação a positividade e o bioma $(\mathrm{p}=0,17)$. Com relação à faixa etária destes indivíduos observamos que a positividade para Blastocystis spp. foi mais elevada no grupo em que a faixa etária dos indivíduos era de 5 a 15 anos do que na população do Grupo II onde os indivíduos apresentavam idade igual ou maior que 16 anos (Tabela 2). Com relação ao sexo indivíduos, não houve diferença significante entre masculino e feminino e os resultados encontrados mostraram que as variáveis sexo e bioma não possuem influência significativa em relação à positividade para Blastocystis spp.

Tabela 2. Percentual de positividade para Blastocystis spp. em relação ao sexo, idade e bioma dos indivíduos participantes da pesquisa nos municípios de Barão de Melgaço, Cáceres, Nova Xavantina e Cuiabá - MT, 2014.

\begin{tabular}{ccccc}
\hline \multicolumn{5}{c}{ Positividade de Blastocystis spp. } \\
\hline Sexo & Positivo & Negativo & Total & Resultado \\
\hline Masculino & $62(8,75 \%)$ & $244(43,46 \%)$ & 306 & $\mathrm{p}=0,15$ \\
Feminino & $65(9,18 \%)$ & $337(47,59 \%)$ & 402 & \\
\hline Faixa etária & Positivo & Negativo & Total & Resultado \\
Grupo I & $79(11,15 \%)$ & $279(39,40 \%)$ & 358 & $\mathrm{p}=0,013$ \\
Grupo II & $48(6,77 \%)$ & $302(42,65 \%)$ & 350 & \\
\hline Bioma & Positivo & Negativo & Total & Resultado \\
Pantanal & $65(9,18 \%)$ & $336(47,48 \%)$ & 401 & $\mathrm{p}=0,17$ \\
Cerrado & $62(8,75)$ & $245(34,60 \%)$ & 307 & \\
\hline
\end{tabular}




\section{Discussão}

O diagnóstico das parasitoses intestinais é realizado por meio de diversas técnicas laboratoriais de exames parasitológicos para análise de fezes. O método de sedimentação espontânea, embora não seja o mais sensível para determinadas espécies, tem demonstrado resultados adequados para Blastocystis spp. (Aguiar et al. 2007, Eymael et al. 2010, Inaba et al. 2016), sendo assim, o presente estudo reflete de forma apropriada a positividade para este protozoário na população pesquisada.

As parasitoses intestinais apresentam frequências elevadas em países em desenvolvimento, como o Brasil, ocorrendo variações quanto à região de cada país, sendo influenciada pelas condições de saneamento básico, nível socioeconômico, graus de escolaridade, idade e os hábitos de higiene dos indivíduos (Machado et al. 1999, Silva-Neto et al. 2010, Zanetti et al. 2020).

Segundo dados do Ministério das Cidades, atual Ministério do Desenvolvimento Regional e também dados apontados pelo Serviço Nacional de Informações sobre Saneamento (SNIS) considerando a série histórica referente ao ano de 2014 dos 141 municípios do Estado, apenas 31 possuíam rede de esgotamento sanitário e 108 cidades mato-grossenses contavam com o serviço de abastecimento de água Tratada (Brasil 2020).

Fatores de riscos como o consumo de água não tratada e cuidados com os animais foram observados nos municípios abordados neste estudo, mais de $85 \%$ dos entrevistados não tinham acesso à rede de esgoto em suas residências, mais de um quarto dos entrevistados utilizavam a água procedente de poços ou cisternas e mais da metade deles tinham animais de estimação em seus quintais.

A positividade de Blastocystis spp. na população geral encontrada neste estudo foi semelhante a encontrada nas amostras fecais analisadas no laboratório de Parasitologia da Faculdade de Medicina de Marilia-SP, onde $16,7 \%$ dos pacientes encontravam-se parasitados pelo protozoário (Martins et al. 2007). Uma meta-analise sobre infecção por Blastocystis spp. no Brasil, demonstrou que a prevalência combinada na população brasileira geral é de $24 \%$, no Centro-Oeste, as maiores taxas foram encontradas nos estados de Mato Grosso do Sul (41,0\%) e Mato Grosso (17,0\%) (Zanetti et al. 2020).

Outros trabalhos demonstraram que em regiões com condições precárias de saneamento básico os índices de positividade tendem a aumentar, em moradores de favelas de Belo Horizonte 22,4\% das amostras de fezes estavam positivas para Blastocystis spp. (Gil et al. 2013), uma pesquisa realizada com população indígena da etnia Tapirapé na Amazônia mato-grossense revelou a presença do parasita em 21\% das amostras analisadas (Malheiros et al. 2011).

Fatores ambientais como condições climáticas podem estar relacionadas a ocorrência de Blastocystis spp., as infecções pelo protozoário são mais comuns durante o tempo quente (Masry et al. 1990, Visser et al. 2011). Tanto no bioma Cerrado quanto no bioma Pantanal as temperaturas tendem a ser elevadas durante a maior parte do ano, fator esse que corrobora para a elevada positividade de Blastocystis spp. nos dois biomas, independentemente do período de coleta.

A análise individual dos municípios evidenciou que a positividade foi mais elevada em Barão de Melgaço, embora apresente uma população relativamente pequena, o município conta com apenas uma unidade básica de saúde para atendimento das populações urbanas e rurais. É provável que o acesso a exames laboratoriais e a ações de educação em saúde realizadas naquela localidade não consiga atender a demanda da população, além disso, o município não dispõe de sistema de 
esgotamento sanitário ligado à rede pública para tratamento de esgoto doméstico, tais fatores podem estar contribuindo para o maior percentual de positividade.

O município de Cáceres embora também não tenha sistema de tratamento de esgoto disponível para a grande maioria dos bairros, possui em sua rede de saúde um número maior de Estratégia da Saúde da Família (ESF) e o acesso a ações preventivas, mesmo que ainda não satisfatórios, são facilitados pelos agentes de saúde, campanhas que visam ações educativas e distribuição de medicamentos para tratamento da população, estas ações podem ter contribuído para a apresentação de uma positividade menor, já que fatores socioeconômicos e socioculturais como a ausência de saneamento básico, falta de educação sanitária, o baixo nível educacional e cultural, baixa renda familiar, precária higienização pessoal, coletiva e do peridomicílio, falta de hábitos higiênicos principalmente no que diz respeito a lavagem das mãos e dos alimentos, contato com animais, água inapropriada para o consumo, contribuem para alta prevalência das parasitoses intestinais (Carvalho e Gomes 2013, Gomes et al. 2016).

O município de Nova Xavantina possui uma rede com cinco ESF no total, porém observouse que quase metade da população participante do estudo (42,5\%) não tem o hábito de realizar algum tratamento prévio da água consumida no domicílio, sendo ingerida diretamente como sai da torneira, fato este que pode estar contribuindo para a alta positividade de Blastocystis spp. nesta localidade. A transmissão de Blastocystis spp. pela água não tratada ou más condições sanitárias foi indicada em outros estudos (Kain 1987; Nimri 1993, ABDULSALAM ET AL. 2013 WAWRZYNIAK ET AL. 2013).

Dados coletados sobre o município de Cuiabá demonstraram que $42,59 \%$ da população que participou da pesquisa, tem o hábito de filtrar a água antes de sua utilização, fato que minimiza a contaminação através da veiculação hídrica.

Os dados obtidos neste estudo em relação à positividade para Blastocystis spp. e o sexo dos indivíduos estão em consonância com outros descritos na literatura (Garcia et al. 1984; Torres et al. 1992, Zanetti et al. 2020), demonstrando não haver diferença significativa entre a positividade e o sexo, indivíduos do sexo masculino e feminino podem ser acometidos por Blastocystis spp. na mesma proporção.

Entretanto, parece haver uma influência da idade dos indivíduos na positividade de Blastocystis spp., a população de maior incidência são crianças menores de 16 anos (Kobayashi et al. 1995). Análise de fezes de crianças de uma creche no município de Botucatu-SP demonstraram que 34,7 $\%$ das amostras eram positivas para Blastocystis spp. (Guimarães e Sogayar 1993) e no município de São Paulo esse índice foi de 38,3\% no ano de 2004 (Amato Neto et al. 2004), outros trabalhos demonstraram que a maior incidências em crianças está relacionada aos hábitos de higiene, acesso ao saneamento básico, contato próximo a animais e informações (Wawrzyniak, 2013, El Safadi et al 2014).

Blastocystis spp. é reconhecido como um dos parasitas intestinais mais prevalentes em crianças, em diversas regiões no mundo (Devera et al. 1998, El Safadi 2014). Os mesmos autores destacam que B. hominis foi o parasita mais frequentemente encontrado em crianças na pré-escola em Ciudad Bolivar, na Venezuela, superando a Entamoeba coli, um protozoário comensal de alta frequência naquele país, assim como Giardia lamblia e os geohelmintos Ascaris lumbricoides e Trichuris trichiura.

Os dados deste trabalho permitem identificar esse público como vulnerável para a incidência do protozoário, nos municípios pesquisados, indicando a possibilidade de transmissão interpessoal 
entre as crianças e por meio de contato com animais, contaminação ambiental ou mesmo a ocorrência de ingestão de alimentos e/ou água contaminados, demonstrando a necessidade da implementação de medidas relacionadas a saneamento básico e programas contínuos de educação sanitária que possibilite melhoria na condição de vida dessas crianças (Uchôa 2001, Carvalho e Gomes 2013, Wawrzyniak et al. 2013).

Embora os resultados obtidos neste estudo não tenham demonstrado significância estatística em relação à positividade de Blastocystis spp. com os biomas Cerrado e Pantanal, sabe-se que a presença desse protozoário pode estar relacionada às condições socioambientais (Lu e Yung 2009), fatores ambientais como a condição climática, tipo de solo, hábitos culturais, juntamente com a qualidade do saneamento básico fornecido e hábitos de higiene das populações são fatores frequentemente associados à exposição e colonização de enteroparasitas (Visser et al. 2011).

Autores destacam a diferença entre os índices de prevalência de Blastocystis ssp. nas diversas populações estudadas pelo mundo, e que estas diferenças tão grandes de uma região para outra sem dúvida obedecem a fatores geográficos e a fatores dependentes do parasita que ainda não se conhece (Devera 1998, Martins et al. 2007, Inaba et al. 2016).

Por ser transmitido por via fecal-oral, tanto por contato direto quanto indiretamente por meio de água e alimentos contaminados por fezes dos indivíduos infectados, torna-se importante conhecer a relação do Blastocystis spp com o meio ambiente para uma maior compreensão de seu ciclo biológico, sua transmissão, distribuição e possível controle.

\section{Conclusão}

Este trabalho possibilitou um maior conhecimento acerca da realidade mato-grossense quanto a infecção por Blastocystis spp., embora este parasita ainda mantenha vários aspectos relativos à sua epidemiologia controversos e a quantidade de estudos ainda permaneça insuficiente, tivemos a oportunidade de observar que o percentual de positividade deste parasita no território matogrossense parece estar muito mais relacionados a faixa etária dos indivíduos. Ressalta-se, ainda, a grande relevância observar que o clima quente nos dois biomas pesquisados, bem como as interações entre os animais e humanos igualmente predominantes nos dois biomas podem de fato contribuir de sobremaneira para as altas taxas de positividade encontradas nesta pesquisa.

Em todo caso, estudos mais específicos acerca deste protozoário tornam-se necessários, a fim de se promover o conhecimento científico e auxiliar no preenchimento das lacunas ainda existentes bem como a inclusão da pesquisa para Blastocystis spp. na rotina diagnóstica dos exames laboratoriais, maior conhecimento acerca de sua epidemiologia, distribuição, sintomatologia bem como melhor padronização terapêutica adequada.

Participação dos autores: WNMZ - coleta e análise das amostras e dos dados, redação do manuscrito; LMM, SLS, SKIC, JJS - análise dos dados e revisão do manuscrito; MFL, MEVS, SSSC, LFB - coleta dos dados e análise das amostras; AFM - coordenador do projeto e análise das amostras e dos dados, redação do manuscrito.

Aprovação ética e licenças: aprovado pelo comitê de ética em pesquisa do Instituto de Ciências Biomédicas da USP sob o parecer no 310.501 . 
Disponibilidade dos dados: as amostras coletadas no decorrer de 2014 das quais resultaram este estudo encontram-se armazenadas sob guarda do Laboratório de Biologia e Parasitologia- LaBPar da Universidade do Estado de Mato GrossoCampus Universitário de Cáceres e disponíveis para consulta e para o desenvolvimento de novos projetos.

Fomento: não houve financiamento de pesquisa.

Conflito de Interesses: Não há conflito de interesses relacionado a este estudo.

\section{Referências}

Abdulsalam AM, Ithoi I, Al-Mekhlafi1 HM, Khan AH, Ahmed A, Surin J, Mak, JW. 2013. Prevalence, predictors and clinical significance of Blastocystis sp. in Sebha, Libya. Parasites \& Vectors, 6 (86): 2-8.

Aguiar JIA, Gonçalves AQ, Sodré FC, Pereira, SR, Bóia, MN, Lemos ERS, Daher, R R. 2007. Intestinal protozoa and helminths among Terena Indians in the State of Mato Grosso do Sul. Rev. Soc. Bras. Med. Trop., 40 (6): 631-634.

Alarcón, RSR, Amato Neto V, Gakiya E, Bezerra RC. 2007. Observações sobre Blastocystis hominis e Cyclospora cayetanensis em exames parasitológicos efetuados rotineiramente. Revista da Sociedade Brasileira de Medicina Tropical, 40 (2): 253-255.

Amato Neto V, Alarcón RSR, Gakiya E, Bezerra RC, Ferreira CS, Braz LMA. 2003. Blastocistose: controvérsias e indefinições. Revista da Sociedade Brasileira de Medicina Tropical, 36: 515-517.

Amato Neto V, Alarcon RSR, Gakiya EG; Ferreira CS, Bezerra RC, Santos AG. 2004. Elevada porcentagem de blastocistose em escolares de São Paulo, SP. Revista da Sociedade Brasileira de Medicina Tropical, 37 (4): 354-356.

Amâncio FAM, Pascotto VM, Souza LR, Calvi SA, Pereira PCM. 2012. Intestinal parasitic infections in HIV/AIDS patients: epidemiological, nutritional and immunological aspects. J Venom Anim Toxins incl Trop Dis, 18:225-35.

Audebert C, Even G, Cian A, Loywick A, Merlin S, Viscogliosi E, Chabé, M. 2016. Colonization with the enteric protozoa Blastocystis is associated with increased diversity of human gut bacterial microbiota. Sci. Rep. 6:25255, doi: 10.1038 / srep25255

Brasil, 2020. Sistema Nacional de Informações sobre Sanamento. Disponivel em: http://app4.mdr.gov.br/serieHistorica/\# Acesso em: 30/07/2020.

Cabrine-Santos M, Cintra EN, Carmo RA, Nascentes GAN, Pedrosa AL, Correia D, Oliveira-Silva MB. 2015. Occurrence of Blastocystis spp. in Uberaba, Minas Gerais, Brazil. Rev. Inst. Med. Trop. Sao Paulo, 57(3):211-215

Carvalho NEDS, Gomes NP. 2013. Prevalência de enteroparasitoses em crianças na faixa etária de 6 a 12 anos na escola pública Melvin Jones em Teresina-PI. R. Interd. 6(4):95-101.

Cheng HS, Haung ZF, Lan WH, Kuo TC, Shin JW. 2006. Epidemiology of Blastocystis hominis and other intestinal parasites in a vietnamese female immigrant population in Southern Taiwan. J. Med. Sci., 22:166-70.

Cimerman S, Ladeira MCT, Iuliano WA. 2003. Blastocistose: nitazoxanida como nova opção. Revista da Sociedade Brasileira de Medicina Tropical, 36 (3): 415-417.

Costanza, R. Valuing Natural Capital and Ecosystem Services toward the Goals of Efficiency, Fairness, and Sustainability. Eco. Services, v.43, 2020.

Devera RA. 1999. Blastocystis hominis parasita intestinal pouco estudado no Brasil. Jornal Brasileiro de Medicina, 76 : 85-89.

Devera RA, Velásquez V J, Velásquez MJ. 1998. Blastocistosis en pre-escolares de Ciudad Bolívar, Venezuela. Cad. Saúde Pública, 14(2):401-407. 
Devera, RA. 1998. Blastocystis hominis: o enigma continua. Revista da Sociedade Brasileira de Medicina Tropical, 31(5):491-493.

El Safadi D, Gaayeb L, Meloni D, Cian A, Poirier P, Wawrzyniak I et al. Children of Senegal river basin show the highest prevalence of Blastocystis sp. ever observed worldwide. 2014. BMC Infect Dis, 14: 164

Eymael D, Schuh GM, Tavares RG. 2010. Padronização do diagnóstico de Blastocystis hominis por diferentes técnicas de coloração. Revista da Sociedade Brasileira de Medicina Tropical, 43 (3): 309-312.

Garcia LS, Bruckner DA, Clancy, MN. 1984. Clinical relevance of Blastocystis hominis. The Lancet, 323 (8388) :12331234.

Gil FF, Barros MJ, Macedo NA, Júnior CG, Redoan R, Busatti H, Gomes MA, Santos JF. 2013. Prevalence of intestinal parasitism and associated symptomatology among hemodialysis patients. Revista do Instituto de Medicina Tropical de São Paulo, 55 (2): 69-74. 2013.

Gomes SCS, Rodrigues SR, Silva AB, Arruda AKS, Silva NM, Macedo RS, Lima ENP, Ferreira IEA. 2016. Educação em saúde como instrumento de prevenção das parasitoses intestinais no município de Grajaú - MA. Pesquisa em Foco, São Luís, 21 (1): 34-45.

Guimarães S and Sogayar MI. 1993. Blastocystis hominis: occurrence in children and staff members of municipal daycare center from Botucatu, São Paulo, Brazil. Mem. Ist. Oswaldo Cruz, 88 (3): 427 - 9.

Inaba MHM, Oliveira CS, Seo NM, Chiu WC, Takizawa MGMH. 2016. Identificação de Blastocystis hominis em amostras coletadas em bairros da região oeste de Cascavel-PR. Revista Varia Scientia, 2 (1): 16-22.

Jelinek T, Peyerl G, Lo Scher T, Von Sonnenburg F, Nothdurft HD. 1997. The role of Blastocystis hominis as a possible intestinal pathogen in travellers. J. Infect., 35:63-66.

Jones, K. E. et al. Global Trends in Emerging Infectious Diseases. Nature, v.451, 2008.

Kain KC, Noble MA, Freeman HJ, Barteluk RL. 1987. Epidemiology and clinical features associated with Blastocystis hominis infection. Diagn. Microbiol. Infect. Dis., 8: 235-244.

Khademvatan S, Masjedizadeh R, Rahim F, Mahbodfar H, Salehi R, Yousefi-Razin E, Foroutan M. 2017. Blastocystis and irritable bowel syndrome: Frequency and subtypes from Iranian patients. Parasitol Int, 66(2):142-145

Kobayashi J, Hasegawa H, Forli AA, Nishimura NF, Yamanaka A, Shimabukuro T, Sato Y. 1995. Prevalence of intestinal parasitic infection in five farms in Holambra, São Paulo, Brazil. Revista do Instituto de Medicina Tropical de Sao Paulo, 37(1):13-18.

Lu CT and Yung YJ. 2009. Epidemiology of Blastocystis hominis and other intestinal parasites among the immigrant population in northeastern Taiwan by routine physical examination for residence approval. Journal Microbioly Immunology and Infection, 2(1):505-509.

Machado, RC, Marcari EL, Cristante SFV, Carareto CMA. 1999. Giardíase e Helmintíases em crianças de creches e escolas de $1^{\circ}$ e $2^{\circ}$ graus (públicas e privadas) da cidade de Mirassol (SP, Brasil). Revista da Sociedade Brasileira de Medicina Tropical, 32 (5): 697-704.

Malheiros, A. F.; Stenvold, C. R.; Clark, C. G.; Braga, G. B.; Shaw, J. J. 2011. Molecular Characterization of Blastocystis spp. obtained from Members of the Indigenous Tapirapé Ethnic Group from the Brazilian Amazon Region, Brazil. American Society of Tropical Medicine and Hygiene, v. 85, n. 6, p. 1050-1053.

Martins LPA, Serapião AATB, Valenciano RF, Pires JEE, Castanho REP. 2007. Frequência de Blastocystis hominis e outras enteroparasitoses em amostras fecais analisadas no laboratório de Parasitologia da Faculdade de Medicina de Marilia-SP. Revista de Parasitologia Tropical, 36 (1): 47-53. 
Masry NA, Bassily S, Farid Z, Aziz AG. 1990. Potential clinical significance of Blastocystis hominis in Egypt. R. Soc. Trop. Med. Hyg, 84:695.

Nascimento AS and Moitinho MLR. 2005. Blastocystis hominis and other intestinal parasites in a community of Pitanga City, Paraná State, Brazil. Rev. Inst. Med. Trop., 47: 213-217.

Nimri LF. 1993. Evidence of an epidemic of Blastocystis hominis infections in preschool children in northern Jordan. J. Clin. Microbiol., 31: 2706-2708.

Nolla AC e Cantos GA. 2005. Relação entre a ocorrência de enteroparasitoses em manipuladores de alimentos e aspectos epidemiológicos em Florianópolis, Santa Catarina, Brasil. Cad. Saúde Pública, 21(2):641-645.

Osman M, Bories J, El Safadi D, Poirel MT, Gantois N, Benamrouz-Vanneste S, et al. 2015. Prevalence and genetic diversity of the intestinal parasites Blastocystis sp. and Cryptosporidium spp. in household dogs in France and evaluation of zoonotic transmission risk. Vet Parasitol, 214, 167-170.

Patz, J. A. et al. Unhealthy Landscapes: Policy Recommendations on Land Use Change and Infectious Disease Emergence. Env. H. Perspec., v.12, 2004.

Silberman JD, Sogin ML, Leipe DD, Clark CG. 1996. Human parasite finds taxonomic home. Nature, 380 (6573):398.

Silva AA. 2006. Incidência de Blastocystis hominis na População da Cidade do Rio de Janeiro, R J. News Lab, $76:$ 86-96.

Stenzel DJ and Boreham, PF. 1996. Blastocystis hominis revisited. Clin. Microbiol. Rev., 9: 563-584.

Takizawa MGMH, Falavigna DLM, Gomes ML. 2009. Enteroparasitosis and their ethnographic relationship to food handlers in a tourist and economic center in Paraná, Southern Brazil. Revista do Instituto de Medicina Tropical de São Paulo, 51: 31-35.

Tan KSW. 2008. New Insights on Classification, Identification, and Clinical Relevance of Blastocystis spp. Clinical Microbiology Reviews, 21(4): 639-665.

Torres P, Miranda JC, Flores L, Riquelme J, Franjola R, Perez J, Auad S, Hermosilla C, Riquelme S. 1992. Blastocystosis and other intestinal protozoan infections in human riverside communities from Valdivia River Basin, Chile. Rev. Inst. Med. Trop., 34:557-564.

Uchôa CMA, Lobo AGB, Bastos OMP, Matos AD. 2001. Parasitoses intestinais: prevalência em creches comunitárias da cidade de Niterói, Rio de Janeiro - Brasil. Rev. Inst. Adolfo Lutz, 60 (2):97-101.

Velásquez V, Caldera R, Wong W, Cermeño G, Fuentes M, Blanco Y, Aponte M, Devera R. 2005. Elevada prevalência de blastocistose em pacientes do Centro de Saúde de Soledad, Estado Anzoátegui, Venezuela. Rev. Soc. Bras. Med. Trop. 38(4):356-357.

Visser S, Giatti LL, Carvalho RAC, Guerreiro JCH. 2011. Estudo da associação entre fatores socioambientais e prevalência de parasitose intestinal em área periférica da cidade de Manaus (AM, Brasil). Cienc Saúde Coletiva, 16(8):3481-92.

Wawrzyniak I, Poirier P, Viscogliosi E, Dionigia M, Texier C, Delbac F, El Alaoui H. 2013. Blastocystis, an unrecognized parasite: na overview of pathogenesis and diagnosis. Therapeutic Advances in Infectious Disease, 1(5) 167-178

Wilkinson, D. A. et al. Habitat Fragmentation, Biodiversity Loss and the Risk of Novel Infectious Disease Emergence. Journal of The Royal Society Interface, v.15, 2018.

Zanetti AS, Malheiros AF, Matos TA, Longhi FG, Moreira LM, Silva SL, Castrillon SKI, Ferreira SMB, Ignotti E, Espinosa OA. 2020. Prevalence of Blastocystis sp. Infection in several hosts in Brazil: a systematic review and meta-analysis. Parasites Vectors, 13:30 https://doi.org/10.1186/s13071-020-3900-2. 
Zhang X, Zhang S, Qiao JY, Wu X, Zhao L, Liu Y, Fan X.2012. Ultrastructural insights into morphology and reproductive mode of Blastocystis hominis. Parasitol Res, 110 (3), 1165-72.

De Carli GA. Parasitologia clínica: seleção de métodos e técnicas de laboratório para o diagnóstico das parasitoses humanas. 2a ed. São Paulo: Atheneu, 2007. 